\title{
Chemically and mechanically engineered flux pinning for enhanced electromagnetic properties of $\mathrm{MgB2}$
}

\begin{abstract}
Tuning electronic- and nanostructure is the critical issue for engineering $\mathrm{MgB} 2$ superconductor toward applications. In this chapter, we introduce a variety of chemical and microstructural control techniques that have been developed to artificially enhance flux pinning strength in the material. The influence of chemical additives and oxygen doping on the formation of nanoprecipitates and superconducting properties is reviewed in Sect. 3.1. Carbon doping effects and methods of introducing carbon using different sources are summarized in Sect. 3.2. Microstructural control via ball milling and mechanical alloying techniques is given in Sect. 3.3.
\end{abstract}

